OPEN ACCESS

Edited by:

Robert Klein,

Icahn School of Medicine at Mount

Sinai, United States

Reviewed by:

Umberto Vespasiani-Gentilucci, Campus Bio-Medico University, Italy

Lanlan Chen,

Jilin University, China

*Correspondence:

Shun-Fa Yang

ysf@csmu.edu.tw

Yung-Po Liaw

Liawyp@csmu.edu.tw

Specialty section:

This article was submitted to Applied Genetic Epidemiology,

a section of the journal

Frontiers in Genetics

Received: 30 November 2021

Accepted: 22 February 2022

Published: 07 March 2022

Citation:

Hsueh K-C, Nfor ON, Hsu S-Y, Yang S-F and Liaw Y-P (2022) Type 2

Diabetes, PNPLA3 rs738409

Polymorphism, and the Risk of Liver

Cirrhosis: Analysis of Taiwan Biobank.

Front. Genet. 13:822700.

doi: 10.3389/fgene.2022.822700

\section{Type 2 Diabetes, PNPLA3 rs738409 Polymorphism, and the Risk of Liver Cirrhosis: Analysis of Taiwan Biobank}

\author{
Kuan-Chun Hsueh ${ }^{1,2}$, Oswald Ndi Nfor ${ }^{3}$, Shu-Yi Hsu ${ }^{3}$, Shun-Fa Yang ${ }^{4,5 *}$ and Yung-Po Liaw ${ }^{3,6 *}$ \\ ${ }^{1}$ Department of Surgery, Tungs' Taichung Metro Harbor Hospital, Taichung, Taiwan, ${ }^{2}$ Department of Post-Baccalaureate \\ Medicine, College of Medicine, National Chung Hsing University, Taichung, Taiwan, ${ }^{3}$ Department of Public Health and Institute of \\ Public Health, Chung Shan Medical University, Taichung, Taiwan, ${ }^{4}$ Institute of Medicine, Chung Shan Medical University, \\ Taichung, Taiwan, ${ }^{5}$ Department of Medical Research, Chung Shan Medical University Hospital, Taichung, Taiwan, ${ }^{6}$ Department \\ of Medical Imaging, Chung Shan Medical University Hospital, Taichung City, Taiwan
}

Type 2 diabetes (T2D) and liver cirrhosis remain significant public health threats in Taiwan. These conditions are reported to be associated with the rs738409 polymorphism of the patatin-like phospholipase domain-containing protein three gene (PNPLA3) in European populations. We assessed the effect of T2D and PNPLA3 rs738409 polymorphism on liver cirrhosis among Taiwan Biobank (TWB) participants. In total, 17,985 participants in TWB had their health records linked to the National Health Insurance Research Database (NHIRD). Participants included those who visited the assessment centers between 2008 and 2015, with an age range between 30 and 70 years of age. We performed logistic regression analysis to investigate the odds ratios (OR) for liver cirrhosis among participants based on the T2D status and rs738409 genotypes. Genotyping was performed using the Axiom Genome-Wide TWB Array Plate. In our analysis, 150 of the 17,619 eligible participants were identified as cirrhosis cases. Based on the univariate analysis, liver cirrhosis was positively associated with $\mathrm{T} 2 \mathrm{D}(\mathrm{OR}, 1.83$; 95\% $\mathrm{Cl} 1.23-2.70)$ whereas, the variant rs738409 was not (regardless of the genetic model). The variant and T2D, however, showed significant interactions in the additive, genotype, and dominant models ( $p$ values of $0.0302,0.0395$, and 0.0455 , respectively). We observed a statistically significant association between T2D and liver cirrhosis and variant rs738409 with an OR of 1.71 (95\% Cl, 1.03-2.84) for individuals carrying a $\mathrm{G}$ allele compared to those with a $\mathrm{C}$ allele and 2.92 (95\% Cl 1.07-7.99) for GG compared to CC individuals. According to our study, Taiwanese adults with T2D and the rs738409 GG genotype are more likely to develop liver cirrhosis.

Keywords: liver, observational study, type 2 diabetes, polymorphism, chronic disease BUN (mg/dl) 0.996 0.954-1.040

\section{INTRODUCTION}

Globally, liver diseases are one of the most important public health issues (Asrani et al., 2019). They remain a leading cause of morbidity and mortality. Cirrhosis is a potentially fatal complication of chronic liver disease, which may manifest $20-40$ years after infection (Smith et al., 2019). It is the 11th leading cause of death globally (Asrani et al., 2019) and has a considerable economic impact (Smith et al., 2019). It is commonly caused by hepatitis, alcoholism, and non-alcoholic liver disease, but other causes remain unknown in approximately 5\%-30\% of those with this condition. 
(Nalbantoglu and Jain, 2019; Patel et al., 2019). As a result, exploring other possible variables may increase our understanding of the disease pathology.

Cirrhosis is strongly linked to viral hepatitis in several countries, including Taiwan (Mokdad et al., 2014; Sepanlou et al., 2020; Yang et al., 2020). In spite of this, the underlying causes and disease burden vary across demographic groups and geographic regions (Sepanlou et al., 2020). Diabetes is among the chronic conditions that have previously been linked to liver cirrhosis and mortality in various ethnic groups (Holstein et al., 2002; Quintana et al., 2016; Goh et al., 2017), even though the underlying pathogenic mechanisms are not entirely clear (Tolman et al., 2007). Mortality risk was found to be higher in patients with compensated liver cirrhosis who had T2D (Quintana et al., 2016).

Aside from the common causes, genetic factors have also been associated with liver cirrhosis (Stickel et al., 2020). However, the association between liver cirrhosis and a number of polymorphic variants has been inconsistent (Basyte-Bacevice et al., 2019; Chen et al., 2020). Among the previously identified polymorphisms, rs738409 variant of the PNPLA3 gene has been strongly associated with cirrhosis and indices of liver damage, especially in Caucasians (Romeo et al., 2010). This variant has also been linked to liver-related events and death among Italians with nonalcoholic fatty liver disease (NAFLD) (Grimaudo et al., 2020), which is becoming more common in the eastern regions of Asia (Zhang et al., 2015). Despite its role in aggravating liver steatosis, the rs738409 variant has also been associated with a decreased risk of T2D in metabolically unhealthy middle-aged and elderly Chinese populations (Xia et al., 2019).

On the contrary, an earlier study found no association between diabetes and the PNPLA3 variant (Speliotes et al., 2010). Of note, diabetes and cirrhosis remain public health concerns in Taiwan. These two conditions have been associated with rs738409 polymorphism mainly in other populations, as cited above. Considering that the rs738409 variant has not been studied extensively among Asians, we examined the effect of this variant in combination with $\mathrm{T} 2 \mathrm{D}$ on liver cirrhosis in TWB participants.

\section{MATERIALS AND METHODS}

\section{Data Sources}

Through the data management system of the Health and Welfare Data Science Center (HWDC), we linked participants' genotype data in TWB to their medical records in the NHIRD using personal identification numbers. Participants included those who visited the assessment centers between 2008 and 2015, with an age range between 30 and 70 years of age. Written informed consent was obtained from each participant before they were assessed. Disease information in the NHIRD was available from 1998 to 2015. Approval was obtained from the Institutional Review Board of Chung Shan Medical University. All authors had access to the study data and had reviewed and approved the final manuscript.

\section{Definition of the Variables}

Data were available for 17,957 subjects in TWB. We excluded those with an incomplete questionnaire $(n=271)$ and genotyping information $(n=27)$, as well as cases of cirrhosis identified before the diagnosis of diabetes $(n=40)$. Finally, we identified 150 participants as cirrhosis cases and 17,469 as control individuals. Liver cirrhosis was diagnosed based on the International Classification of Diseases, Ninth Revision, Clinical Modification (ICD-9-CM), codes 571.2, 571.5, 571.6, 572.2, $572.3,572.4,572.8,573.0)$ and either one-time hospitalization or two outpatient visits. Detailed information on the diagnoses of T2D has been described elsewhere (Nfor et al., 2018).

\section{Genotyping}

The PNPLA3 rs738409 was genotyped using the custom Taiwan Biobank chip Axiom $^{\mathrm{TM}}$ Genome-Wide Array Plate System (Affymetrix, Santa Clara, CA, United States). The SNP call rate was $>95 \%$ and was in Hardy-Weinberg equilibrium ( $p$-value $>1.0 \times$ $10^{-3}$. The minor allele frequency (MAF) was $>0.05$.

\section{Statistical Analysis}

Our data analyses were conducted with the software package PLINK 1.09 and the SAS software (version 9.4). We compared baseline parameters using the $\mathrm{x}^{2}$ test. We investigated the effects of T2D and PNPLA3 rs738409 genetic variant on liver cirrhosis based on univariate and multivariate logistic regression analyses. The additive, dominant, and recessive models were used to determine the allele and genotype-specific ORs. Adjusted variables included sex, age, educational level, smoking, alcohol intake, body mass index (BMI), physical activity, hepatitis $\mathrm{C}$ virus ( $\mathrm{HCV})$, hepatitis $\mathrm{B}$ virus (HBV), and hyperlipidemia. In order to reduce potential bias, patients with cirrhosis before the diagnosis of diabetes were excluded from the final logistic regression models. We tested for interaction by including the term $\mathrm{rs} 738409^{*} \mathrm{~T} 2 \mathrm{D}$ in the regression models. The ORs were estimated along with their 95\% CI.

\section{RESULTS}

Table 1 describes the general characteristics of study participants. Overall, 150 of the 17,469 eligible participants were identified as cirrhosis cases. Among the cases of cirrhosis, 60 were of the rs738409 CC genotype, 62 were of the CG genotype, and 28 were of the GG genotype. About $21.33 \%(n=32)$ of participants with liver cirrhosis had T2D. According to the univariate analysis, liver cirrhosis was positively associated with T2D (OR, 1.83; 95\% CI 1.23-2.70) but the variant rs738409 was not despite the genetic model used (Table 2). Multivariate analyses, however, found no significant correlation between cirrhosis and rs738409 or T2D regardless of the model used. There was, however, a significant interaction between the variant and T2D in the additive, genotype, and dominant models (with $p$ values of 0.0302, 0.0395 , and 0.0455 , respectively).

According to the univariate analyses, $\mathrm{HCV}$ and $\mathrm{HBV}$ infections both contributed significantly to cirrhosis risk, with ORs of 6.89 (95\% CI, 4.3-11.05) and 4.19 (95\% CI, 2.98-5.88), respectively. In spite of the analysis model, both variables remained significantly 
TABLE 1 | Basic data of study participants.

\begin{tabular}{|c|c|c|c|}
\hline & No liver cirrhosis & Liver cirrhosis & $p$-value \\
\hline & N (\%) & N (\%) & \\
\hline PNPLA3 rs738409 & & & 0.2504 \\
\hline $\mathrm{CC}$ & 6,487 (37.13) & $60(40.00)$ & \\
\hline CG & 8,329 (47.68) & $62(41.33)$ & \\
\hline GG & 2,653 (15.19) & $28(18.67)$ & \\
\hline Sex & & & 0.0004 \\
\hline Female & $9,049(51.80)$ & $56(37.33)$ & \\
\hline Male & $8,420(48.20)$ & $94(62.67)$ & \\
\hline Age, year & & & $<0.0001$ \\
\hline 30-39 & 4,932 (28.23) & $17(11.33)$ & \\
\hline $40-49$ & 4,627 (26.49) & $33(22.00)$ & \\
\hline $50-59$ & $4,752(27.20)$ & $58(38.67)$ & \\
\hline 60-70 & 3,158 (18.08) & $42(28.00)$ & \\
\hline Education & & & 0.0454 \\
\hline Elementary School & $1,002(5.74)$ & $8(5.33)$ & \\
\hline Junior/Senior High School & 6,776 (38.79) & $73(48.67)$ & \\
\hline University and above & $9,691(55.48)$ & $69(46.00)$ & \\
\hline Cigarette smoking & & & 0.0001 \\
\hline No & 13,217 (75.66) & $93(62.00)$ & \\
\hline Yes & $4,252(24.34)$ & $57(38.00)$ & \\
\hline Alcohol drinking & & & 0.0007 \\
\hline No & 15,681 (89.76) & $122(81.33)$ & \\
\hline Yes & $1788(10.24)$ & $28(18.67)$ & \\
\hline Body Mass Index $\left(\mathrm{kg} / \mathrm{m}^{2}\right)$ & & & 0.6506 \\
\hline$<18.5$ and 18.5-24 & 8,927 (51.10) & $71(47.34)$ & \\
\hline $24-27$ & 5,027 (28.78) & 47 (31.33) & \\
\hline$\geq 27$ & $3,515(20.12)$ & $32(21.33)$ & \\
\hline Exercise & & & 0.0184 \\
\hline No & 10,280 (58.85) & 74 (49.33) & \\
\hline Yes & 7,189 (41.15) & $76(50.67)$ & \\
\hline $\mathrm{HCV}$ & & & $<0.0001$ \\
\hline Negative & 17,066 (97.69) & $129(86.00)$ & \\
\hline Positive & $403(2.31)$ & $21(14.00)$ & \\
\hline HBV & & & $<0.0001$ \\
\hline Negative & 15,504 (88.75) & $98(65.33)$ & \\
\hline Positive & 1965 (11.25) & $52(34.67)$ & \\
\hline T2D & & & 0.0023 \\
\hline No & 15,209 (87.06) & 118 (78.67) & \\
\hline Yes & $2,260(12.94)$ & 32 (21.33) & \\
\hline Hyperlipidemia & & & $<0.0001$ \\
\hline No & $12,229(70.00)$ & $81(54.00)$ & \\
\hline Yes & $5,240(30.00)$ & $69(46.00)$ & \\
\hline
\end{tabular}

T2D, type 2 diabetes; $H C V$, hepatitis $C$ virus; $H B V$, hepatitis $B$ virus.

associated with liver cirrhosis as shown in Table 2. For subjects aged $40-49,50-59$, and $60-70$, the ORs and 95\% CIs were respectively 2.07 (1.15-3.72), 3.54 (2.06-6.09), and 3.86 (2.19-6.79) when compared with those aged 30-39. Our analysis of cases with and without T2D based on rs738409 genotypes (Table 3) indicated that diabetic individuals with the $\mathrm{G}$ allele had a higher cirrhosis risk compared to those with the C allele (OR, 1.71; 95\% CI, 1.03-2.84), as did diabetic individuals with the GG genotype compared to those carrying the CC genotype (OR, 2.92; 95\% CI, 1.07-7.99).

\section{DISCUSSION}

Using the data of TWB participants collected from 2008 to 2015, we found that the PNPLA3 gene variant rs738409 was not associated with liver cirrhosis while type 2 diabetes was.
Despite these, we observed a significant interaction between the variant and $\mathrm{T} 2 \mathrm{D}$ as shown in the additive, genotype, and dominant models. Furthermore, our analyses showed that rs738409 GG individuals who had T2D were more prone to liver cirrhosis than their CC counterparts.

Taiwan still faces a substantial number of liver diseases, including cirrhosis. It should be noted that cirrhosis is more prevalent among people with viral hepatitis (Mokdad et al., 2014). In contrast, $12.3-57 \%$ of patients with cirrhosis were also diagnosed with T2D (Trombetta et al., 2005). In a recent review, Lee and colleagues claimed that there is a higher prevalence of diabetes in patients with liver cirrhosis than previously believed (Lee et al., 2019). Numerous studies have already suggested a link between liver cirrhosis and T2D. However, there is little data about genetic links between these two health conditions. To gain more insight into the relationship, 
TABLE 2 | Association of liver cirrhosis with PNPLA3 rs738409 and T2D.

\begin{tabular}{|c|c|c|c|c|c|c|}
\hline \multirow[t]{2}{*}{ PNPLA3 rs738409 } & & \multirow{2}{*}{$\begin{array}{c}\text { Univariate } \\
\text { OR } \\
(95 \% \mathrm{Cl})\end{array}$} & \multirow{2}{*}{$\frac{\text { Additive model }}{\text { aOR }(95 \% \mathrm{Cl})}$} & \multirow{2}{*}{$\frac{\text { Genotype model }}{\text { aOR }(95 \% \mathrm{Cl})}$} & \multirow{2}{*}{$\frac{\text { Dominant model }}{\text { aOR }(95 \% \mathrm{Cl})}$} & \multirow{2}{*}{$\frac{\text { Recessive model }}{\text { aOR }(95 \% \mathrm{Cl})}$} \\
\hline & & & & & & \\
\hline Additive model & G vs. C & $1.01(0.80-1.28)$ & $1.04(0.82-1.31)$ & - & - & - \\
\hline \multirow[t]{2}{*}{ Genotype model } & CG vs. CC & $0.81(0.56-1.15)$ & - & $0.83(0.58-1.18)$ & - & - \\
\hline & GG vs. CC & $1.14(0.73-1.79)$ & - & 1.19 (0.76-1.88) & - & - \\
\hline Dominant model & $G G+C G$ vs. CC & 0.89 (0.64-1.23) & - & - & $0.91(0.66-1.27)$ & - \\
\hline Recessive model & GG vs. $C G+C C$ & $1.28(0.85-1.94)$ & - & - & - & $1.32(0.87-2.01)$ \\
\hline T2D & Yes vs. No & $1.83(1.23-2.70)$ & $1.11(0.72-1.71)$ & $1.12(0.72-1.73)$ & $1.11(0.72-1.71)$ & $1.11(0.72-1.72)$ \\
\hline Sex & Male vs. Female & 1.80 (1.29-2.52) & 1.33 (0.89-1.99) & 1.32 (0.88-1.98) & 1.33 (0.89-2.00) & $1.32(0.88-1.98)$ \\
\hline \multicolumn{7}{|l|}{ Age (years) } \\
\hline 40 to 49 & 40-49 vs. 30-39 & 2.07 (1.15-3.72) & 1.7 (0.93-3.09) & 1.69 (0.93-3.07) & $1.70(0.94-3.09)$ & 1.69 (0.93-3.07) \\
\hline 50 to 59 & 50-59 vs. 30-39 & 3.54 (2.06-6.09) & $2.76(1.54-4.93)$ & $2.76(1.54-4.94)$ & $2.76(1.54-4.95)$ & $2.75(1.54-4.92)$ \\
\hline 60 to 70 & 60-70 vs. 30-39 & 3.86 (2.19-6.79) & 3.07 (1.63-5.80) & 3.05 (1.62-5.76) & 3.08 (1.63-5.80) & $3.06(1.62-5.77)$ \\
\hline \multicolumn{7}{|l|}{ Education } \\
\hline Junior/Senior High School & ref: Elementary School & $1.35(0.65-2.81)$ & $1.98(0.93-4.21)$ & $1.95(0.91-4.15)$ & $1.97(0.93-4.20)$ & $1.96(0.92-4.18)$ \\
\hline University and above & ref: Elementary School & $0.89(0.43-1.86)$ & $1.63(0.75-3.53)$ & $1.61(0.74-3.49)$ & $1.63(0.75-3.53)$ & $1.62(0.75-3.50)$ \\
\hline Cigarette smoking & Yes vs. No & $1.91(1.37-2.65)$ & $1.40(0.93-2.10)$ & $1.41(0.94-2.12)$ & $1.40(0.93-2.10)$ & $1.41(0.94-2.12)$ \\
\hline Alcohol drinking & Yes vs. No & $2.01(1.33-3.04)$ & $1.31(0.83-2.09)$ & $1.31(0.82-2.08)$ & $1.31(0.83-2.09)$ & $1.31(0.83-2.09)$ \\
\hline \multicolumn{7}{|l|}{ Body Mass Index (kg/m²) } \\
\hline$<18.5$ & ref: 18.5 to 24 & $0.23(0.03-1.65)$ & $0.30(0.04-2.20)$ & $0.29(0.04-2.14)$ & $0.30(0.04-2.20)$ & $0.30(0.04-2.16)$ \\
\hline 24 to 27 & ref: 18.5 to 24 & $1.12(0.77-1.63)$ & $0.89(0.60-1.30)$ & $0.89(0.60-1.30)$ & $0.88(0.60-1.30)$ & $0.89(0.61-1.30)$ \\
\hline$\geq 27$ & ref: 18.5 to 24 & $1.09(0.72-1.66)$ & $0.94(0.60-1.45)$ & $0.93(0.60-1.45)$ & $0.93(0.60-1.45)$ & $0.94(0.60-1.45)$ \\
\hline Exercise & Yes vs. No & $1.47(1.07-2.03)$ & $1.13(0.80-1.60)$ & $1.13(0.80-1.59)$ & $1.13(0.80-1.59)$ & $1.13(0.80-1.60)$ \\
\hline $\mathrm{HCV}$ & Positive vs. Negative & $6.89(4.3-11.05)$ & 5.87 (3.60-9.57) & $5.79(3.55-9.46)$ & $5.83(3.57-9.51)$ & $5.86(3.59-9.56)$ \\
\hline HBV & Positive vs. Negative & $4.19(2.98-5.88)$ & $4.55(3.21-6.44)$ & $4.56(3.22-6.46)$ & $4.54(3.21-6.43)$ & $4.56(3.22-6.46)$ \\
\hline Hyperlipidemia & Yes vs. No & $1.99(1.44-2.75)$ & $1.39(0.96-2.01)$ & $1.39(0.96-2.01)$ & $1.39(0.97-2.01)$ & $1.39(0.96-2.00)$ \\
\hline$p$-value for interaction rs738409*T2D & - & 0.0302 & 0.0395 & 0.0455 & 0.1872 & \\
\hline
\end{tabular}

The ORs with their 95\% Cls were estimated by a logistic regression model. The aORs with their 95\% Cls were estimated by a multiple logistic regression model.

TABLE 3 | Association of liver cirrhosis with rs738409 genotypes in individuals with and without T2D.

\begin{tabular}{|c|c|c|c|}
\hline \multirow[t]{2}{*}{ PNPLA3 rs738409 } & & \multirow{2}{*}{$\begin{array}{c}\text { No T2D } \\
\text { aOR }(95 \% \mathrm{Cl})\end{array}$} & \multirow{2}{*}{$\frac{\text { T2D }}{\text { aOR }(95 \% \mathrm{Cl})}$} \\
\hline & & & \\
\hline Additive model & G vs. C & $0.90(0.69-1.18)$ & $1.71(1.03-2.84)$ \\
\hline \multirow[t]{2}{*}{ Genotype model } & CG vs. CC & $0.71(0.47-1.06)$ & $1.63(0.69-3.87)$ \\
\hline & GG vs. CC & $0.95(0.56-1.60)$ & $2.92(1.07-7.99)$ \\
\hline Dominant model & $G G+C G$ vs. $C C$ & $0.77(0.53-1.11)$ & $1.92(0.85-4.33)$ \\
\hline Recessive model & $G G$ vs. $C G+C C$ & $1.13(0.69-1.84)$ & $2.17(0.95-4.95)$ \\
\hline
\end{tabular}

The aORs with their $95 \% \mathrm{Cl}$ s were estimated using multiple logistic regression models after controlling for sex, age, education, cigarette smoking, alcohol drinking, BMI, exercise, HBV, HCV, and hyperlipidemia.

we replicated the rs738409 variant and showed that it may interact with $\mathrm{T} 2 \mathrm{D}$ to increase cirrhosis risk. This variant showed associations with liver disease, T2D, and other disorders in diverse populations as mentioned above. Additionally, it has been linked to non-viral hepatitisassociated hepatocellular carcinoma among T2D patients (Ueyama et al., 2016).

The relationship between viral hepatitis and liver cirrhosis is well known. Our univariate and multivariate models also showed that participants with HBV and HCV infection had an increased risk for cirrhosis. Cirrhosis is believed to develop in most viral hepatitis patients around the age of 65 or so (Pradat et al., 2007). Our study found that cirrhosis prevalence was higher among individuals aged 50 to 59 and 60 to 70 .
Our study has the advantage of a large sample size. By using personal identification numbers, we were able to link genetic, clinical, and lifestyle information from the TWB database to medical records from the NHIRD; these data enabled more detailed and accurate analyses at the individual level. Our study was limited, however, by the lack of information regarding the severity of liver cirrhosis. There is a need for further research into the role of T2D and PNPLA3 rs738409 in the development of liver cirrhosis.

This study indicates that Taiwanese adults with T2D who have the PNPLA3 rs738409GG genotype might have a higher risk for liver cirrhosis than those with the CC genotype. Findings like these contribute to a better understanding of the genetic bases of liver cirrhosis, which may be useful in personalized medicine.

\section{DATA AVAILABILITY STATEMENT}

The original contributions presented in the study are included in the article/Supplementary Material, further inquiries can be directed to the corresponding authors.

\section{ETHICS STATEMENT}

The study protocol followed the guidelines for human studies and was conducted ethically in accordance with the World Medical Association Declaration of Helsinki. Ethical approval was 
obtained from the Institutional Review Board of Chung Shan Medical University. Participants provided written informed consent before being assessed.

\section{AUTHOR CONTRIBUTIONS}

$\mathrm{K}-\mathrm{CH}, \mathrm{ON}, \mathrm{S}-\mathrm{YH}, \mathrm{S}-\mathrm{FY}$, and Y-PL conceived and designed the study. S-YH, ON, and Y-PL analyzed and interpreted data. $\mathrm{K}-\mathrm{CH}$ drafted the manuscript with support from S-FY. ON, S-YF, and Y-PL critically reviewed the manuscript for important

\section{REFERENCES}

Asrani, S. K., Devarbhavi, H., Eaton, J., and Kamath, P. S. (2019). Burden of Liver Diseases in the World. J. Hepatol. 70 (1), 151-171. doi:10.1016/j.jhep.2018. 09.014

Basyte-Bacevice, V., Skieceviciene, J., Valantiene, I., Sumskiene, J., Petrenkiene, V., Kondrackiene, J., et al. (2019). TM6SF2 and MBOAT7 Gene Variants in Liver Fibrosis and Cirrhosis. Ijms 20 (6), 1277. doi:10. 3390/ijms20061277

Chen, V. L., Chen, Y., Du, X., Handelman, S. K., and Speliotes, E. K. (2020). Genetic Variants that Associate with Cirrhosis Have Pleiotropic Effects on Human Traits. Liver Int. 40 (2), 405-415. doi:10.1111/liv.14321

Goh, G. B.-B., Pan, A., Chow, W.-C., Yuan, J.-M., and Koh, W.-P. (2017). Association between Diabetes Mellitus and Cirrhosis Mortality: the Singapore Chinese Health Study. Liver Int. 37 (2), 251-258. doi:10.1111/liv. 13241

Grimaudo, S., Pipitone, R. M., Pennisi, G., Celsa, C., Cammà, C., Di Marco, V., et al. (2020). Association between PNPLA3 Rs738409 C>G Variant and LiverRelated Outcomes in Patients with Nonalcoholic Fatty Liver Disease. Clin. Gastroenterol. Hepatol. 18 (4), 935-944. doi:10.1016/j.cgh.2019.08.011

Holstein, A., Hinze, S., Thiessen, E., Plaschke, A., and Egberts, E.-H. (2002). Clinical Implications of Hepatogenous Diabetes in Liver Cirrhosis. J. Gastroenterol. Hepatol. 17 (6), 677-681. doi:10.1046/j.1440-1746.2002. 02755.x

Lee, W. G., Wells, C. I., McCall, J. L., Murphy, R., and Plank, L. D. (2019). Prevalence of Diabetes in Liver Cirrhosis: A Systematic Review and MetaAnalysis. Diabetes Metab. Res. Rev. 35 (6), e3157. doi:10.1002/dmrr.3157

Mokdad, A. A., Lopez, A. D., Shahraz, S., Lozano, R., Mokdad, A. H., Stanaway, J., et al. (2014). Liver Cirrhosis Mortality in 187 Countries between 1980 and 2010: a Systematic Analysis. BMC Med. 12 (1), 145. doi:10.1186/s12916-014-0145-y

Nalbantoglu, I., and Jain, D. (2019). "Cryptogenic Cirrhosis: Old and New Perspectives in the Era of Molecular and Genomic Medicine," in Seminars in Diagnostic Pathology (St Louis: Elsevier) 36 (6), 389-394.

Nfor, O. N., Wu, M.-F., Lee, C.-T., Wang, L., Liu, W.-H., Tantoh, D. M., et al. (2018). Body Mass index Modulates the Association between CDKAL1 Rs10946398 Variant and Type 2 Diabetes Among Taiwanese Women. Sci. Rep. 8 (1), 13235. doi:10.1038/s41598-018-31415-4

Patel, N., Sharma, B., and Samant, H. (2019). "Cryptogenic Cirrhosis," in: StatPearls. Treasure Island (FL): StatPearls Publishing.

Pradat, P., Voirin, N., Tillmann, H. L., Chevallier, M., and Trépo, C. (2007). Progression to Cirrhosis in Hepatitis C Patients: an Age-dependent Process. Liver Int. 27 (3), 335-339. doi:10.1111/j.1478-3231.2006.01430.x

Quintana, J. O., García-Compean, D., González, J. A., Pérez, J. Z., González, F. J., Espinosa, L. E., et al. (2016). The Impact of Diabetes Mellitus in Mortality of Patients with Compensated Liver Cirrhosis-A Prospective Study. Ann. Hepatol. 10 (1), 56-62. doi:10.1016/S1665-2681(19)31588-1

Romeo, S., Sentinelli, F., Dash, S., Yeo, G. S. H., Savage, D. B., Leonetti, F., et al. (2010). Morbid Obesity Exposes the Association between PNPLA3 I148M (Rs738409) and Indices of Hepatic Injury in Individuals of European Descent. Int. J. Obes. 34 (1), 190-194. doi:10.1038/ijo.2009.216

Sepanlou, S. G., Safiri, S., Bisignano, C., Ikuta, K. S., Merat, S., Saberifiroozi, M., et al. (2020). The Global, Regional, and National burden of Cirrhosis by Cause intellectual content. All authors approved the final version of the manuscript.

\section{FUNDING}

This work was supported by Chung Shan Medical University and Tungs' Taichung Metro Habor Hospital (grant number, CSMUTTM-107-01) and the Ministry of Science and Technology of Taiwan (MOST 109-2121-M-040-002 and MOST 110-2121-M040-002).

in 195 Countries and Territories, 1990-2017: a Systematic Analysis for the Global Burden of Disease Study 2017. Lancet Gastroenterol. Hepatol. 5 (3), 245-266. doi:10.1016/S2468-1253(19)30349-8

Smith, A., Baumgartner, K., and Bositis, C. (2019). Cirrhosis: Diagnosis and Management. Am. Fam. Physician 100 (12), 759-770.

Speliotes, E. K., Butler, J. L., Palmer, C. D., Voight, B. F., Hirschhorn, J. N., Crn, N., et al. (2010). PNPLA3variants Specifically Confer Increased Risk for Histologic Nonalcoholic Fatty Liver Disease but Not Metabolic Disease. Hepatology 52 (3), 904-912. doi:10.1002/hep.23768

Stickel, F., Lutz, P., Buch, S., Nischalke, H. D., Silva, I., Rausch, V., et al. (2020). Genetic Variation in HSD17B13 Reduces the Risk of Developing Cirrhosis and Hepatocellular Carcinoma in Alcohol Misusers. Hepatology 72 (1), 88-102. doi:10.1002/hep.30996

Tolman, K. G., Fonseca, V., Dalpiaz, A., and Tan, M. H. (2007). Spectrum of Liver Disease in Type 2 Diabetes and Management of Patients with Diabetes and Liver Disease. Diabetes care 30 (3), 734-743. doi:10.2337/ dc06-1539

Trombetta, M., Spiazzi, G., Zoppini, G., and Muggeo, M. (2005). Review Article: Type 2 Diabetes and Chronic Liver Disease in the Verona Diabetes Study. Aliment. Pharmacol. Ther. 22, 24-27. doi:10.1111/j.1365-2036.2005.02590.x

Ueyama, M., Nishida, N., Korenaga, M., Korenaga, K., Kumagai, E., Yanai, H., et al. (2016). The Impact of PNPLA3 and JAZF1 on Hepatocellular Carcinoma in Non-viral Hepatitis Patients with Type 2 Diabetes Mellitus. J. Gastroenterol. 51 (4), 370-379. doi:10.1007/s00535-015-1116-6

Xia, M.-F., Lin, H.-D., Chen, L.-Y., Wu, L., Ma, H., Li, Q., et al. (2019). The PNPLA3 Rs738409 C>G Variant Interacts with Changes in Body Weight over Time to Aggravate Liver Steatosis, but Reduces the Risk of Incident Type 2 Diabetes. Diabetologia 62 (4), 644-654. doi:10.1007/s00125-018-4805-x

Yang, T.-W., Wang, C.-C., Tsai, M.-C., Wang, Y.-T., Tseng, M.-H., and Lin, C.-C. (2020). Comorbidities and Outcome of Alcoholic and Non-alcoholic Liver Cirrhosis in Taiwan: A Population-Based Study. Ijerph 17 (8), 2825. doi:10. 3390/ijerph17082825

Zhang, L., You, W., Zhang, H., Peng, R., Zhu, Q., Yao, A., et al. (2015). PNPLA3 Polymorphisms (Rs738409) and Non-alcoholic Fatty Liver Disease Risk and Related Phenotypes: a Meta-Analysis. J. Gastroenterol. Hepatol. 30 (5), 821-829. doi:10.1111/jgh.12889

Conflict of Interest: The authors declare that the research was conducted in the absence of any commercial or financial relationships that could be construed as a potential conflict of interest.

Publisher's Note: All claims expressed in this article are solely those of the authors and do not necessarily represent those of their affiliated organizations, or those of the publisher, the editors and the reviewers. Any product that may be evaluated in this article, or claim that may be made by its manufacturer, is not guaranteed or endorsed by the publisher.

Copyright (C) 2022 Hsueh, Nfor, Hsu, Yang and Liaw. This is an open-access article distributed under the terms of the Creative Commons Attribution License (CC BY). The use, distribution or reproduction in other forums is permitted, provided the original author(s) and the copyright owner(s) are credited and that the original publication in this journal is cited, in accordance with accepted academic practice. No use, distribution or reproduction is permitted which does not comply with these terms. 